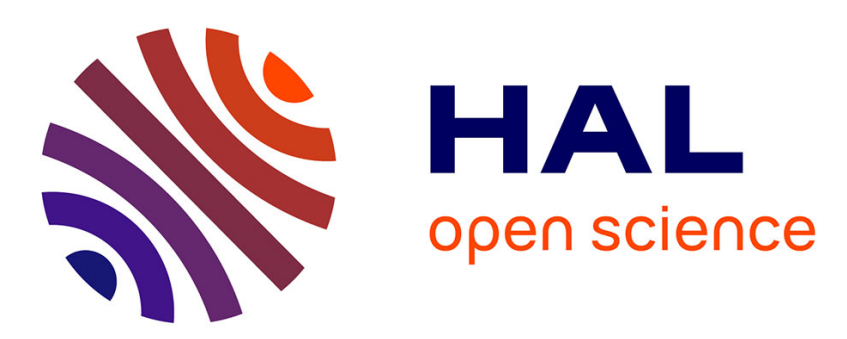

\title{
Les relations économie-histoire et le statut scientifique des sciences sociales chez Hicks et Schumpeter
}

\author{
Daniel Dufourt
}

\section{To cite this version:}

Daniel Dufourt. Les relations économie-histoire et le statut scientifique des sciences sociales chez Hicks et Schumpeter. Revue Française d'Economie, 1992, Volume 7 (Numéro 1), pp.167 - 214. halshs00394581

\section{HAL Id: halshs-00394581 https://shs.hal.science/halshs-00394581}

Submitted on 19 Jun 2009

HAL is a multi-disciplinary open access archive for the deposit and dissemination of scientific research documents, whether they are published or not. The documents may come from teaching and research institutions in France or abroad, or from public or private research centers.
L'archive ouverte pluridisciplinaire HAL, est destinée au dépôt et à la diffusion de documents scientifiques de niveau recherche, publiés ou non, émanant des établissements d'enseignement et de recherche français ou étrangers, des laboratoires publics ou privés. 


\title{
Les relations économie- histoire et le statut scientifique des sciences sociales chez Hicks et Schumpeter
}

\author{
Daniel DUFOURT
}

«La recherche des fondements d'une méthodologie des sciences sociales est la tâche la plus importante de ce temps dans le domaine de la théorie de la connaissance. ${ }^{1}$ »" Carl Menger.

Le caractère récurrent des rencontres entre économistes et historiens ${ }^{2}$ conduit à s'interroger sur les circonstances qui, dans l'évolution de la pratique scientifique des deux disciplines concernées, produisent la nécessité de telles rencontres et leurs enjeux théoriques. Plusieurs démarches concurrentes peuvent légitimement prétendre éclaircir les raisons de la persistance de ce phénomène et du caractère occasionnel de ses manifestations.

Il est tout d'abord possible, dans une perspective de sociologie de la science ${ }^{3}$ de mettre l'accent sur l'impact décisif en matière d'orientation des recherches, des modalités institutionnelles retenues pour introduire l'enseignement de l'économie politique dans les cursus universitaires et d'en analyser les effets sur la conceptualisation des relations entre analyse économique et investigation historique. A ne s'en tenir qu'à l'économie politique, on observera avec C Gide ([1890], [1895], [1907], [1908]) que l'existence, en France, de deux pôles de la pensée économique, à savoir l'Institut et les Facultés de Droit a durablement infléchi l'activité théorique dans le sens d'une mise à l'écart de l'enseignement universitaire de l'économie, tant de l'économie mathématique que des aspects les plus féconds de la «querelle des méthodes»: «l'école de Vienne n'a trouvé en France presqu'aucun adepte, bien moins encore que l'école d'Eisenach» [1908], XVI, p. 25. En effet, autour de l'Institut et de la Société d'Economie Politique, se regroupent les membres de l'école libérale française qui « a exercé une influence énorme pendant près d'un siècle sur la littérature économique de la France» (ibidem, p. 7). Or, cette école libérale reste réfractaire au psychologisme qu'elle décèle dans l'école de Vienne. Quant aux responsables des enseignements d'économie politique dans les facultés de droit, bien qu'ayant pu bénéficier au cours de leur formation d'une initiation aux travaux de l'école historique allemande du droit (voir Savigny), grâce notamment à l'enseignement de P. Gide, ils apparaissent comme très sensibles, à l'instar de leurs collègues Scandinaves ${ }^{4}$; à l'influence des idées interventionnistes en matière de politique sociale qui émane des socialistes de la chaire, mais ont « peine à admettre le caractère de relativité que l'école allemande attribue à tous les phénomènes économiques», Gide [1908], p. 25. La réflexion méthodologique semble dès lors durablement accaparée par E. Durkheim, qui cite et

\footnotetext{
1 "Die Begründung einer Methodologie der Sozialwissenschaften ist die wichtigste Aufgabe der Gegenwart auf dem Gebiet der Erkenntnistheorie“, C.Menger [1889], p.489.

${ }^{2}$ Cf. par exemple les numéros 1, [1950] ; 1, [1965] et 2, [1991] de la Revue économique, ainsi que les tomes 54 [1964], supplément, et 75, $\mathrm{n}^{\circ} 2$ [1985] de $\Gamma$ American economic review.

${ }^{3}$ Classique et en quelque sorte «pré-kuhnienne», cf. P.B. Lecuyer [1978]

${ }^{4}$ Cf. P. Falbeck [1908] Die volkswirtschaftliche Literatur Skandinaviens im 19. Jahrhundert in Mélanges
} Schmoller vol. I, XIX, pp. 1-15 
commente longuement G. Schmoller, et plus encore par ses disciples F. Simiand et M. Halbwachs. De sorte que la première rencontre entre économistes et historiens s'effectuera en France par l'entremise de la sociologie durkheimienne.

L'école historique allemande a exercé une influence beaucoup plus décisive sur la formation des universitaires américains, à l'origine sans doute de la prépondérance du courant institutionnaliste aux Etats-Unis dans les années vingt et trente.

D'une part, comme le montre P. R. Senn 5 [1989], p. 263, les fondateurs de l' " American Economic Association» créèrent des statuts si inspirés de ceux du «Verein fur Sozialpolitik», et témoignant d'un biais si prononcé en faveur de l'intervention de l'Etat, qu'ils durent par la suite être modifiés. Cet épisode n'a rien d'étonnant si l'on en croit H.W. Farnam, qui, au terme d'une enquête auprès de 126 économistes et sociologues enseignant aux EtatsUnis et au Canada, dont il estime qu'ils représentent la très grande majorité du corps professoral de ces disciplines, constate que 59 d'entre eux ont effectué leurs études en Allemagne, 20 y ayant obtenu leur doctorat, la durée moyenne des séjours dans ce pays étant de 2 ans (Farnam, [1908], p. 26). Le premier à avoir inauguré cette «filière» allemande de formation est J.B. Clark en 1873. Il sera suivi, pour s'en tenir aux auteurs les plus connus, par Simon Patten [1876], R.T. Ely [1877], E.R. Seligman et F. Taussig [1879], J. Laurence Laughlin [1891], F. A. Fetter [1893].

En sens inverse, l'immigration dans les années trente et quarante d'économistes formés ou influencés par l'école de Vienne, tels O. Morgenstern, J. Schumpeter, L. von Mises, va contribuer de manière déterminante au relatif déclin du courant institutionnaliste aux EtatsUnis.

La récurrence des rencontres entre économistes et historiens peut s'interpréter ensuite en référence au jeu des acteurs, à leur stratégie dans cette institution sociale que représente une discipline scientifique établie. Il est tentant, d'autant que l'intéressé lui-même a joué ${ }^{6}$ et joue encore ${ }^{7}$ un rôle non négligeable dans la récurrence de ces rencontres, d'appliquer la méthodologie de la «rhétorique économique» élaborée par D.N. McCloskey à l'analyse de ces stratégies d'acteurs. Dans l'évolution d'une discipline scientifique, la recherche de "nouvelles alliances" peut apparaître comme un élément décisif de succès dans la lutte concurrentielle que se livrent les protagonistes des divers programmes de recherche en présence. En France, on remarquera par exemple l'accent mis par A. Marchal en 1950, sur «les émulations et emprunts réciproques» qui s'établissent entre les deux disciplines, dès lors que la reconnaissance de l'économie comme science de l'homme oblige à une réévaluation des notions de causalité et de déterminisme, et à une intégration du temps historique et des facteurs d'irréversibilité dans l'analyse. Avec le recul du temps, l'ample recension des problèmes de méthode à laquelle s'est livré A. Marchal apparaît comme une tentative très affirmée de préserver un héritage difficilement constitué face au retour offensif de l'école néoclassique. Plus récemment, R. Boyer [1989], après avoir dressé le constat des impasses auxquelles conduit l'allégeance à des théories et philosophies économiques peu enclines à l'analyse des processus historiques et indifférentes aux problèmes conceptuels que soulève l'émergence de règles et de comportements nouveaux, formule une sorte de manifeste en

\footnotetext{
${ }^{5}$ Curieusement l'enquête quasi exhaustive de l'auteur sur l'influence de G. Schmoller sur la littérature économique et historique de langue anglaise ne mentionne pas l'article de Farnam auquel il est fait référence cidessous.

${ }^{6}$ Cf. D. N. Mc Closkey [1976] , pp. 434- 461.

${ }^{7}$ Cf. D. N. Mc Closkey [1986], pp. 63- 69.
} 
faveur d'une conception élargie de la dynamique économique, intégrant l'analyse des régimes d'accumulation dans une théorie des transformations structurelles et du changement institutionnel. De même, aux Etats-Unis, à la faveur de la discussion organisée sous les auspices de l'American Economie Association en 1985 sur le thème : «l'histoire économique, condition nécessaire mais non suffisante de la formation de l'économiste», P. David est amené à plaider en faveur d'une étude systématique des caractères essentiellement historiques des processus dynamiques en économie.

Du côté des historiens, on observera d'une part l'effort de J. Bouvier en vue d'inscrire l'héritage de l'école des Annales, à la faveur duquel l'histoire a pu s'identifier à «une projection des sciences sociales dans le passé» (F. Mauro), dans le cadre rigoureux d'un mariage ("brassage chimique", plutôt que "mélange mécanique") intentionnel des deux modes d'analyse, économique et historique. On sera également sensible, d'autre part, au débat formulé en termes d'opposition de générations qu'a suscité, dans l'histoire économique anglosaxonne, l'introduction des méthodes quantitatives (R. Andreano [1970]). L'enjeu scientifique de ce débat, comme l'observe justement G. Barraclough [1980] déborde largement les frontières de l'histoire économique, puisqu'il s'agit de savoir s'il est légitime «au moins dans les cas favorables, de construire une situation fictive (counterfactual), grâce à laquelle on peut mesurer le décalage entre ce qui s'est réellement produit et ce qui aurait pu se produire dans des circonstances différentes» (Barraclough [1980], p. 128).

La limite d'une investigation en termes de «rhétorique économique» réside dans son incapacité à rendre compte des enjeux théoriques des changements dans les stratégies d'acteurs qui constituent, dans cette perspective, le facteur essentiel de l'évolution d'une discipline. Aussi nous inscrivons-nous dans une troisième démarche pour tenter d'expliquer la nécessaire récurrence des rencontres entre économistes et historiens. Il s'agit de se situer à un niveau épistémologique et de montrer que selon le type de conception relative aux formes de la connaissance en économie, une articulation déterminée des relations entre l'économie et l'histoire en constitue le sous-produit inéluctable. Dans cette perspective, deux traditions apparaissent comme largement dominantes : celle du positivisme, qui, sur la base d'une classification des connaissances affirme l'unité de la méthode scientifique et récuse dans ce domaine toute spécificité aux sciences sociales. La projection de l'économie sur le matériau historique débouche sur un genre déterminé de théorie structuraliste de l'histoire. L'axe Walras-Hicks nous semble le mieux traduire cette première tradition. La deuxième tradition, issue en partie du débat allemand sur la spécificité des sciences de l'esprit, débouche en économie sur une théorie de la relation comportement-institutions qui, à son tour, nécessite une autre forme d'articulation des deux modes d'analyse, économique et historique. L'axe Menger-Schumpeter est incontestablement le plus représentatif de cette deuxième tradition. Après avoir illustré les analyses historiques auxquelles conduisent ces deux traditions nous montrerons en quoi l'existence de problèmes non résolus, relatifs à leurs fondements épistémologiques respectifs, produit la nécessité récurrente d'une rencontre entre économistes et historiens.

\section{Les enjeux de deux lectures théoriques de l'histoire : rationalité marchande versus représentations collectives chez Hicks et Schumpeter}


Après avoir rappelé comment la naissance de l'économie marchande est assimilée par J. Hicks à une discontinuité majeure dans le cours de l'histoire des sociétés, nous montrerons comment, à la thèse de l'essence économique des discontinuités historiques, Schumpeter oppose celle d'une continuité historique d'essence sociologique. La différence de point de vue est ici liée, comme nous le montrerons dans une deuxième partie, outre à une conceptualisation différente des relations entre dynamique économique et évolution historique, au type de représentation des phénomènes sociaux qui résulte de la reconnaissance ou non de la pertinence de la distinction entre sciences de la nature et sciences de l'esprit et aux effets de cette distinction sur la recherche des fondements de la méthodologie des sciences sociales.

\section{Histoire d'une transformation}

Soucieux de montrer que l'apparition de l'économie marchande est conforme à une évolution «normale», c'est-à- dire correspondant en moyenne aux besoins essentiels qui se font jour, Hicks s'interroge sur les types d'organisation économique qui ont pu précéder le marché. Paradoxalement, pour mettre en évidence les caractéristiques structurelles de ces organisations, il se réfère à l'entreprise, qu'il considère «dans sa structure interne comme une organisation sans marché». J. Hicks [1973], p. 19. A partir de l'entreprise, il est possible de mettre en évidence deux types d'organisations : l'organisation coutumière et l'organisation autoritaire. La première correspond à une situation d'équilibre constamment renouvelée, et dont la permanence est essentiellement due à la routine, et à l'existence d'un système de règles, la coutume, qui assigne à chacun ses fonctions. La seconde découle d'une évolution de l'économie coutumière sous la pression d'événements extérieurs tels que les guerres, ou simplement l'accroissement démographique. Une économie coutumière ne peut, en effet, fonctionner, étant donné la grande autonomie laissée à chacun que si «les ordres qu'un niveau peut donner à un autre sont étroitement délimités» (Ibidem, p. 21.) Mais que survienne une situation dont ce type d'organisation n'a pas l'expérience et tout le fonctionnement du système est paralysé. La seule issue possible est alors un ajustement de l'ensemble de l'organisation coutumière, dont l'initiative revient à un pouvoir central. Mais la réalisation d'un tel ajustement nourrit de lui-même la disparition de l'organisation coutumière au profit de l'organisation autoritaire. Hicks montre alors que l'organisation autoritaire est le résultat d'un processus inéluctable de bureaucratisation. Processus inéluctable parce que le pouvoir central doit imposer son autorité dans un système qui est à l'origine un système coutumier. Pour imposer son autorité, le pouvoir central doit créer une caste de fonctionnaires. Mais la créer ne suffit pas, il faut la renforcer par trois moyens : la création d'un corps spécial chargé du contrôle et de la surveillance des autres fonctionnaires, l'institution d'un système de promotion, gage de mobilité, et l'établissement d'un système de recrutement qui assure à la fois la permanence de la bureaucratie et son renouvellement indispensable pour que l'autorité du pouvoir central ne soit pas confisquée par une caste héréditaire.

Qu'il s'agisse des économies coutumières ou des économies autoritaires, voire despotiques, «elles reposent toutes sur la notion centrale de prélèvement : impôt, redevance, tribut ou rente foncière (car, en l'absence d'un marché, on ne distingue pas ces termes l'un de l'autre) payé par le paysan ou le cultivateur, le producteur de denrées alimentaires, à une autorité reconnue.» (J. Hicks [1973], op. cit., p. 31). L'organisation de ce prélèvement est à l'origine de la division du travail qui est donc antérieure à l'économie marchande. La division du travail se manifeste ici par une spécialisation fonctionnelle qui s'étend dès que le pouvoir central est en mesure de réaliser une concentration de la "demande". 


\section{Un conflit de rationalités: économie coutumière et activité commerciale}

Ayant caractérisé les deux formes d'organisation économique antérieures au marché, Hicks s'interroge sur les conditions qui ont permis sinon favorisé l'apparition de ce dernier. Une première condition est évidente : il faut qu'apparaisse, dans le cadre de la spécialisation fonctionnelle évoquée, une activité particulière qui est celle du marchand. Or, l'exercice d'une activité commerciale suppose la constitution d'un capital. Sur ce point, Hicks se montre très évasif se bornant à dire que le capital nécessaire à l'activité du marchand n'a pu être constitué que de manière illicite. Hicks s'interroge ensuite sur les conditions qui ont favorisé l'apparition d'un commerce régulier : deux possibilités sont alors évoquées, les rassemblements à l'occasion des fêtes religieuses et les ambassades qui sont la première manifestation d'un commerce inter royal. Dans les deux cas, il s'agit d'un commerce exercé à la frontière de l'économie autoritaire ou coutumière qui ne peut se maintenir que si la constitution d'une classe de marchands est possible, et si les problèmes nés de l'exercice de l'activité commerciale sont résolus. Le premier problème est antérieur logiquement au second : Hicks ne le résoudra pas, considérant probablement que tant l'économie coutumière que l'économie autoritaire tirent des avantages spécifiques de l'activité commerciale, qui est ainsi tolérée. Quant au second problème, il conduit à reconnaître deux nécessités : la protection de la propriété et le contrat. La protection de la propriété est une condition vitale pour le développement de l'activité commerciale : pour que l'échange soit possible, il faut que soit reconnu le droit de propriété de l'échangiste sur la marchandise dont il se défait. De même, la conclusion d'accords contractuels permet seule de transformer une activité commerciale intermittente en une activité permanente : à la livraison immédiate se substitue une promesse de livraison et l'échange devient ainsi un échange de promesses.

Quelles sont les conditions économiques favorables à l'institution d'un droit de propriété et à l'exercice d'une activité fondée sur la conclusion d'accords contractuels? Dans le cadre de l'organisation économique coutumière et autoritaire, la seule possibilité d'introduction de ce nouveau droit de propriété repose sur la confusion de l'activité artisanale et commerciale. Or l'activité artisanale comme l'activité commerciale sont d'autant plus intenses qu'elles concernent des populations davantage concentrées. L'Etat- cité est donc le fer de lance de la pénétration de l'économie marchande dans l'organisation coutumière et autoritaire. Pourquoi l'Etat-cité n'a-t-il fait son apparition qu'en Europe et non en Asie? A cette question essentielle puisqu'elle met à l'épreuve toute sa théorie de l'histoire économique, Hicks n'apporte qu'une réponse qui s'apparente semble-t-il à une stratégie d'esquive : "la cité" européenne est un cadeau de la Méditerranée» ( Hicks [1973], op. cit., p. 47).

Constatons donc l'émergence de l'Etat-cité en Europe et interrogeons- nous avec Hicks sur les ressorts de son développement. L'Etat-cité apparaît d'abord comme une réponse à une manifestation de la loi des rendements décroissants dans l'activité commerciale. En effet, pour un commerce donné, on constate, le volume des échanges étant limité par le niveau de développement des forces productives, une tendance au nivellement des marges commerciales par suite de l'apparition de nouveaux marchands. La diversification de l'activité commerciale est donc un moyen de rétablir les marges de profit et, dans cette perspective, l'Etat-cité offre le maximum d'avantages. Il réalise une concentration de la population dont les besoins entraînent des échanges intenses avec l'extérieur. Mais alors la prospérité de l'Etat-cité dépend de la régularité de ces échanges et de la sûreté de ses approvisionnements. L'Etat-cité s'avère ainsi, dans un second temps, comme le moteur d'un processus de colonisation, les colonies apparaissant d'abord sous la forme de comptoirs avant de devenir des colonies de peuplement. Arrivé à ce stade de sa reconstitution historique de la genèse de l'économie marchande, Hicks 
observe une pause qui lui permet de décrire l'âge d'or que représente la phase de transition entre une société coutumière à son déclin et une économie marchande en voie de formation :

"Ce moment, où l'expansion s'arrête, peut être un moment merveilleux. Les profits sont toujours élevés, mais il ne faut plus les investir dans la poursuite de l'expansion pour les conserver. Une fois cette condition acceptée, on dispose de la richesse et de la sécurité. Que pourrait-on rêver de mieux? La place du marché, autrefois si agitée, a été ramenée à l'ordre. Chacun a sa place dans la société, place à laquelle il lui faut rester, mais qui est réservée et protégée des indiscrétions d'autrui. Par l'intermédiaire des corporations et autres associations du même genre, on peut expérimenter de nouvelles formes de rapports humains. C'est presque une Utopie socialiste . » (J. Hicks [1973], op. cit. p. 67).

Hélas, cet âge d'or est menacé par les conditions mêmes qui lui ont donné naissance.

\section{Les transformations des fonctions de l'Etat sous l'effet de la rationalité marchande}

En effet, à la première phase du développement de l'économie marchande constituée par le système des cités, va succéder une phase où l'existence des centres commerciaux va dépendre d'un Etat à la fois plus étendu dans son ressort géographique que l'Etat-cité, et différent dans son organisation et son fonctionnement. C'est qu'en effet, la pénétration de l'économie marchande dans l'organisation coutumière a profondément modifié les fonctions et les conditions d'exercice du pouvoir central. Ces transformations ont pour origine le développement de la circulation monétaire, l'institution d'un nouveau droit et l'apparition du crédit. La frappe des monnaies devient petit à petit une prérogative royale : d'une part le sceau du roi dispose d'une plus large recevabilité, d'autre part le contrôle de l'émission de la monnaie est vite l'enjeu d'une compétition pour l'exercice du pouvoir politique. Pour ce qui concerne le droit, Hicks observe que les cités " possédaient un droit du commerce issu du droit romain bien que son caractère commercial fût beaucoup plus explicite qu'au temps des Romains» (Ibidem, p. 80). L'intérêt de ce droit, du point de vue du pouvoir central, c'est qu'il repose sur des évaluations exprimées en termes monétaires dont l'Etat pourra faire usage pour déterminer l'assiette de l'impôt. Enfin, l'activité commerciale des cités avait introduit, non seulement le prêt à intérêt entre commerçants et non- commerçants, mais aussi le recours aux premiers intermédiaires financiers. Le développement des contrats d'assurance nourrit l'extension de l'activité bancaire, activité à laquelle l'Etat prête une grande attention, étant donné l'insuffisance chronique des recettes fiscales. Hicks montre qu'incapable d'imposer le revenu, parce que ce concept renvoie à un niveau de développement économique beaucoup plus avancé, l'Etat impose la propriété. Mais l'évaluation des biens possédés étant très difficile, la collecte peu sûre, l'Etat se présente constamment comme un agent emprunteur. Au départ, il n'a guère de crédit, et il en a même d'autant moins qu'il se livre à de nombreuses manipulations monétaires. Tout change lorsque le contrôle de l'Etat sur l'offre de monnaie devient complet : alors, «il n'y a plus de danger que l'Etat refuse de payer une dette exprimée dans sa propre monnaie, puisqu'il lui est toujours possible d'emprunter au système bancaire ; les banques ne peuvent refuser de lui prêter, puisqu'elles peuvent toujours créer de la monnaie pour financer leurs prêts» (J. Hicks, op. cit., p. 106).

\section{L'assujettissement de l'agriculture et de l'industrie au marché}

Les conséquences de la pénétration de l'économie marchande dans l'organisation coutumière ou autoritaire ne sont pas limitées aux fonctions et au fonctionnement de l'Etat. 
Elles affectent également l'agriculture et l'industrie. La pénétration de l'économie marchande dans l'agriculture comporte deux étapes : une pénétration commerciale et une pénétration financière. La pénétration commerciale oblige le seigneur à commercialiser le surplus agricole et surtout à faire reconnaître par des actes juridiques ses droits sur la terre et ceux qui la travaillent. Alors seulement il pourra l'hypothéquer ou la céder. La pénétration financière correspond à une séparation désormais strictement établie entre la propriété et l'exploitation. Il apparaît alors deux systèmes : le fermage, qui oblige le propriétaire à investir des capitaux à long terme dans l'exploitation s'il veut être assuré d'une rente croissante, assimilée ici «au revenu normal d'un investissement commercial» (ibidem, p. 129), et l'exploitation indépendante. Les paysans sont, en effet, les meilleurs acheteurs de terre. Toutefois, ils sont la plupart du temps contraints d'aliéner leur indépendance financière, ne pouvant à la fois acquérir les terres et dégager les ressources nécessaires au financement des investissements. Alors, «l'Etat, qui avait déjà hérité du seigneur une fonction de protection (...) peut aisément fournir des capitaux à l'agriculture au nom du développement» (Ibid., p. 130).

L'apparition de l'industrie suppose la constitution d'un marché du travail. Ce qui caractérise l'ouvrier, c'est qu'il «accomplit une tâche pour quelqu'un d'autre» (Ibid., p. 132). Pour Hicks, ce n'est pas l'économie marchande qui a entraîné la disparition de l'esclavage. En effet, l'esclavage est un système d'approvisionnement en main-d'œuvre tout à fait compatible avec l'exercice d'une activité commerciale. Le marché du travail n'est pas alors le marché du service du travail mais le marché du facteur de production lui-même. Ce qu'il importe donc d'expliquer, aux yeux de Hicks, ce sont les conditions économiques qui ont fait que le marché des esclaves est apparu comme un système d'approvisionnement en main-d'œuvre moins efficient que celui représenté par le marché des services du travail. Ces conditions ont trait aux coûts d'opportunité de l'esclave par rapport au travailleur libre. Dès que l'entretien de l'esclave apparaît comme une dépense relativement onéreuse, le recours au travailleur libre est préférable, mais cette condition n'est pas suffisante, et Hicks reconnaît lui- même son échec : il explique l'apparition d'un marché du travail au sens moderne par le tarissement des sources d'esclaves, c'est-à-dire par un accident historique.

Condition préalable à l'établissement de l'industrie, la disponibilité d'une main-d'œuvre abondante et bon marché reste en définitive inexpliquée dans l'analyse de Hicks. Celui-ci propose d'ailleurs une définition très restrictive du phénomène de révolution industrielle dont l'analyse clôt son ouvrage. La révolution industrielle se caractérise selon cet auteur par l'élargissement du nombre et la variété des capitaux fixes, qui suppose une grande abondance de capitaux liquides et le développement des machines-outils qui entraîne une baisse du prix des capitaux fixes en longue période. Comme Hicks est soucieux de montrer, qu'en dépit d'une phase d'ajustement, la révolution industrielle " a été hautement favorable en fin de compte au salaire réel de la main-d'œuvre», (J. Hicks [1973], op. cit., p. 158), il lui faut justifier la nécessité d'une baisse du prix des capitaux fixes qui a permis de remplacer les premières machines par «une nouvelle génération moins chère et plus efficace» (Ibidem, p. 164), donc d'accroître les profits «sans qu'une épargne additionnelle fût nécessaire» (Ibid. p. 164). Dès lors, l'accumulation de capital a pu donner naissance à de nouvelles activités permettant la résorption du surplus de main-d'œuvre «libéré» par les machines à l'étape précédente, et la hausse des salaires réels. Hicks fonde son argumentation sur ce point sur une interprétation de l'analyse de Ricardo dans le sens d'une théorie de la compensation.

\section{Une lecture «hicksienne» de l'histoire?}


Hicks insiste fortement au début de son ouvrage sur le caractère de sa démarche qui consiste à appliquer quelques idées générales, tirées de la théorie économique, à l'analyse historique (p. 11). Malheureusement, il ne dit rien ou presque sur le contenu de ces idées générales. L'une de celles-ci a trait aux raisons pour lesquelles l'économie marchande a réussi à s'imposer. Pour Hicks, la réussite de cette transformation historique que représente l'avènement de l'économie marchande est due à la diffusion et au partage des gains de l'activité commerciale. Ces gains sont d'ailleurs souvent d'une nature telle qu'ils échappent à l'analyse historique : « Le premier but d'une expansion commerciale n'est pas d'accroître le nombre des biens disponibles, mais de les redistribuer pour les rendre plus utiles. La variété de biens disponibles s'accroît, ce qui entraîne une amélioration des moyens d'existence. C'est un avantage que «l'histoire économique quantitative», dont le travail s'effectue sur des chiffres et des indices de revenu réel, n'est pas à même de mesurer ni même de décrire.» (Ibidem, p. $65)$.

Quels enseignements retirer de cette lecture hicksienne de l'histoire ? Tout d'abord, Hicks associe l'apparition et le développement de l'économie marchande aux courants d'échanges internationaux et à la colonisation. Ensuite, il montre avec une grande clarté les mutations structurelles qui ont permis la pénétration de l'économie marchande dans l'agriculture et l'industrie : ces mutations ont trait aux rapports de propriété et aux rapports sociaux. Dans l'agriculture, le servage fait place au fermage; dans l'industrie l'esclave fait place au travailleur salarié. Néanmoins, la pesanteur sociale et notamment les caractéristiques de l'organisation coutumière de l'économie font que cette évolution normale est, sinon entravée, du moins fortement ralentie par le type de division du travail que greffe l'activité commerciale sur la spécialisation fonctionnelle antérieure. Ce type de division du travail s'exprime par la constitution des corporations qui détiennent le monopole de la formation d'une main-d'œuvre qualifiée. Dès lors, et c'est peut-être sur ce point que Hicks se sépare le plus de Marx, l'économie marchande est impuissante à elle seule à promouvoir un développement de type capitaliste. La démonstration de Hicks est jusque là souvent vraisemblable sinon convaincante. Toutefois, la révolution industrielle et le développement du mode de production capitaliste qui l'accompagne, restent totalement inexpliqués chez Hicks. Les seuls arguments avancés ont trait à l'apparition en quelque sorte miraculeuse des machines, dont les effets seuls permettent cette accumulation sur grande échelle que nécessite l'industrialisation. Ainsi, d'une part l'accumulation ne serait point une nécessité de l'économie marchande, et d'autre part, l'avènement du capitalisme se ramènerait à un accident historique. On découvre ainsi la faiblesse du modèle théorique sous-jacent à l'ouvrage : quel type de développement économique l'économie marchande aurait-elle suscité en l'absence de la révolution industrielle? Les rares indications qui figurent dans l'ouvrage ne permettent pas d'apporter une réponse précise à cette question. Toutefois, il semble bien que Hicks voie dans le développement du système bancaire une transformation profonde qui, révolution industrielle ou pas, aurait modifié profondément la nature de l'économie marchande et donné naissance à une forme capitaliste déterminée d'organisation de l'économie.

F. Perroux ${ }^{8}$ adresse à la théorie de l'histoire de Hicks deux critiques essentielles. La première consiste à rechercher quelle est la justification d'une théorie qui ne s'intéresse qu'au seul marché. Deux hypothèses sont possibles : soit l'économie se réduit au seul marché, soit il n'y a aucune autre théorie de l'économie que celle du marché. Selon F. Perroux, c'est cette dernière conception qui animerait la démarche de Hicks. Mais alors, et nous avons là la deuxième critique essentielle de F. Perroux, peut-on considérer que l'élément moteur de

\footnotetext{
${ }^{8}$ 8. F. Perroux [1973] : Pouvoir et Economie, Bordas, Paris.
} 
l'apparition et du développement de l'économie de marché, c'est-à-dire le principe de l'« All Round Advantage», correspond à l'expérience historique ?

\section{Les racines de l'expansion de l'état national selon J. Schumpeter}

Après avoir rappelé la conception schumpétérienne des rapports entre l'économie et l'histoire, nous exposerons sa théorie de l'impérialisme. Nous nous interrogerons ensuite sur la validité des thèses de Schumpeter au regard de l'expérience historique récente.

Dans sa «Théorie de l'évolution économique» Schumpeter énonce une conception très précise des rapports entre l'économie et l'histoire'. Cette conception est en quelque sorte un sous-produit nécessaire de sa tentative d'élaboration d'une théorie dynamique de l'activité économique. Il convient donc d'indiquer brièvement les fondements de cette théorie. Par circuit, Schumpeter entend un mode de fonctionnement des activités économiques dans lequel un certain état du système économique se reproduit à l'identique. Le circuit est donc l'équivalent de l'équilibre général walrasien à une différence près, importante au demeurant. Tandis que l'équilibre général de Walras est une situation dans laquelle l'entrepreneur ne fait ni bénéfice ni perte, le circuit schumpétérien est l'image d'un état de l'économie où la fonction d'entrepreneur n'existe pas. Donc le circuit schumpétérien ne connaît ni capital, ni capitalistes, ni intérêts. Il se borne à transcrire les relations existantes entre les facteurs originaires : travail et terre. Pour passer du circuit à l'évolution économique, il n'existe logiquement dans le modèle de Schumpeter qu'une seule possibilité et qui consiste «en une combinaison nouvelle des facteurs de production.» L'évolution économique est donc toute entière dépendante d'une fonction qui consiste à réaliser cette combinaison nouvelle des facteurs et qui est l'apanage de l'entrepreneur. Le revenu de l'entrepreneur, le profit, est ainsi clairement rattaché à une activité essentielle puisque d'elle et d'elle seule dépend l'évolution économique. Etudier les motivations de l'entrepreneur et les conditions dans lesquelles il exerce son activité, c'est donc énoncer la logique du capitalisme et, comme nous le verrons, cette logique est pour Schumpeter étrangère à tout esprit de conquête. Mais, caractériser économiquement la fonction de l'entrepreneur et les conséquences de son exercice, c'est se limiter à énoncer une théorie dynamique de l'économie.

Si l'on veut rendre compte de l'évolution historique, il faudra situer l'entrepreneur dans son milieu, et notamment dans les conditions sociales ("l'ambiance") qui définissent les modalités concrètes selon lesquelles la fonction d'entrepreneur peut être exercée. L'histoire en tant que discipline est ainsi réputée théorie de l'environnement. Ce n'est pas à elle d'énoncer les nécessités internes du système économique qui le conduisent à évoluer. De même l'économiste est impuissant à rendre compte des transformations dans les rapports sociaux qui altèrent la logique du système économique. Néanmoins, l'histoire concrète, réelle, est une : tenter de la saisir, c'est tenter de déterminer une hiérarchie des facteurs explicatifs de l'évolution historique. Cette hiérarchie ne met pas, le plus souvent, le facteur économique au premier plan comme Schumpeter essaie de le montrer dans son essai sur l'impérialisme.

La thèse centrale de Schumpeter est que le système économique capitaliste n'a aucun intérêt économique essentiel à une expansion impérialiste : " on peut poser en principe que, partout où règne le libre-échange, aucune classe sociale n'a intérêt à l'expansion militaire» ${ }^{10}$.

\footnotetext{
${ }_{9}^{9}$ J. Schumpeter [1935] Théorie de l'évolution économique, Dalloz, Paris, pp. 305-317
} 
Cette thèse est toute entière subordonnée, ainsi que le fait observer J.C. Passeron ${ }^{11}$, à deux définitions "aussi transhistoriques l'une que l'autre»" ${ }^{12}$. La première définition est celle de l'impérialisme et la seconde celle du capitalisme. L'impérialisme est selon Schumpeter «la disposition, dépourvue d'objectifs, que manifeste un Etat à l'expansion par la force, au-delà de toute limite définissable» ${ }^{13}$. Quant au capitalisme, c'est l'univers de l'entrepreneur, du calcul économique rationnel et donc de la concurrence et du libre échange.

La démarche suivie par Schumpeter, pour rendre compte du phénomène impérialiste dans l'histoire, est très révélatrice : il commence son étude par l'Angleterre du XIXe siècle, où le système capitaliste est le plus développé. Il montre que, si le terme impérialisme a été utilisé en Grande-Bretagne, la réalité qu'il recouvre n'existait pas. L'impérialisme n'a été que le slogan d'une politique intérieure, utilisé pour détourner ses partisans des fins réelles qu'une telle politique eût supposées. Si, en Grande-Bretagne, le terme existait mais non la chose ellemême, c'est l'inverse que l'on constate en remontant le cours de l'histoire. En effet, l'impérialisme est apparu dès l'Antiquité. Partout où il s'est manifesté, ajoute Schumpeter, il a manifesté un caractère générique, qui interdit donc de le rattacher à «l'évolution économique des sociétés modernes ${ }^{14}$. Ce caractère est générique parce qu'il répond aux exigences fondamentales d'une certaine forme d'organisation politique et d'une certaine structure sociale. Ainsi Schumpeter met en évidence dans l'Antiquité l'impérialisme des nations guerrières, dont le ressort peut être soit populaire, soit religieux. Dans tous les cas, l'impérialisme s'avère lié à la présence d'une caste de guerriers, qui suscite les guerres pour justifier son existence. L'impérialisme religieux correspond notamment, selon Schumpeter, au cas arabe: «c'est le prophète des cavaliers nomades qui proclama la guerre sans fin, et non pas n'importe quel prophète ${ }^{15}$. La religion aurait répondu ici à l'attente d'une certaine structure sociale menacée dans son existence. Le peuple des Francs offre, quant à lui, l'exemple d'un impérialisme populaire lié à une disposition en quelque sorte atavique à la conquête.

Après avoir évoqué un impérialisme culturel grec, et l'impérialisme de Rome qui peut seulement s'expliquer par une analyse «des intérêts de classe qui s'y trouvaient engagés» ${ }^{16}$, Schumpeter aborde l'étude de l'impérialisme dans la monarchie absolue des temps modernes. Cette étude joue un rôle central dans sa thèse puisqu'elle va lui fournir les arguments permettant de caractériser l'impérialisme contemporain comme l'expression de la survivance de structures sociales antérieures. Schumpeter, après une minutieuse analyse historique, explique qu'il faut chercher la clé de cet impérialisme dans des nécessités qui tiennent à la structure sociale et, plus particulièrement, aux habitudes héréditaires de la classe nobiliaire, bien plus que dans les avantages concrets que le pouvoir aurait pu attendre de ces conquêtes $\rangle^{17}$. Au terme de cet impressionnant survol historique, Schumpeter peut aborder le problème qui motive la rédaction de l'essai, à savoir les rapports entre impérialisme et capitalisme. Schumpeter conduit alors son argumentation en trois temps : il montre d'abord les résistances qui existent dans une société où domine le système économique capitaliste à d'éventuelles politiques d'agression ; il établit ensuite d'un point de vue théorique l'absence

\footnotetext{
${ }^{10}$ J. Schumpeter [1919] : Contribution à une sociologie des impérialismes in J. Schumpeter [1972] Impérialisme et classes sociales, Editions de Minuit, Paris, p. 123

${ }^{11}$ J.-C. Passeron [1972] Présentation in J. Schumpeter [1972] Impérialisme et classes sociales, Editions de Minuit, Paris, p. 123

${ }^{12}$ J.-C. Passeron [1972], p.23, note 17.

${ }^{13}$ J. Schumpeter [1919], étude citée p. 44

${ }^{14}$ J. Schumpeter [1919], étude citée p. 64

${ }^{15}$ Ibidem, p. 81

${ }^{16}$ Ibid., p. 95

${ }^{17}$ Ibid., p. 103
} 
d'intérêt économique pour la classe des entrepreneurs d'une politique impérialiste, il s'interroge enfin sur les structures sociales qui permettent aujourd'hui l'adoption d'une politique impérialiste.

Sur le premier point, Schumpeter n'a pas de mal à montrer l'existence dans les sociétés capitalistes : d'une opposition des libéraux aux guerres coloniales, de partis ouvertement pacifistes, d'une idéologie socialiste dans la classe ouvrière qui fait qu'« impérialisme socialiste ou impérialisme prolétarien sont choses encore plus inconcevables qu'un impérialisme paysan ${ }^{18}$. Sur le second point, Schumpeter se borne à rappeler les arguments économiques habituels en faveur du libre échange. Il montre également que les seules couches intéressées à la guerre d'un point de vue économique sont constituées des entrepreneurs des industries d'armement qui peuvent effectivement «prendre rang parmi les alliés des forces sociales qui perpétuent les vraies tendances impérialistes.»1 ${ }^{19}$.

Sur le dernier point, Schumpeter met en évidence ce qui constitue à ses yeux une dénaturation de la logique du système capitaliste. La politique protectionniste de la fin du xix ${ }^{\text {ème }}$ siècle et le développement des cartels sont à l'origine de l'apparition d'intérêts économiques favorables à une expansion impérialiste qui, sans eux, n'aurait pas existé. Quels sont donc les facteurs qui expliquent la constitution d'un «capitalisme de monopole» ${ }^{20}$ que Schumpeter dénonce avec la dernière énergie ? Il faut d'abord montrer que le système économique capitaliste ne tend pas, au terme d'une évolution nécessaire, vers l'impérialisme. Schumpeter éprouve les plus grandes difficultés à le faire : comme réconcilier la politique coloniale, le dumping ou exportation monopoliste, le développement de la grande entreprise, avec le caractère irénique de la libre concurrence et du libre échange? Néanmoins, Schumpeter pense y être parvenu en expliquant que ces déviations par rapport à la logique du système capitaliste résultent de ce que la greffe capitaliste a été faite sur un corps social profondément marqué par une pratique séculaire de politiques de conquêtes; en d'autres termes, le régime de la monarchie absolue. Alors, en quelques pages, qui ne manquent pas de grandeur, Schumpeter va dénoncer la collusion du nationalisme et du militarisme, qui prend appui sur «la mentalité des milieux ruraux» ${ }^{21}$ qui, perpétue dans la société capitaliste des orientations politiques anciennes $\rangle^{22}$. L'impérialisme moderne est la conséquence tardive d'une cristallisation des forces précapitalistes «que l'Etat monarchique réorganisa, en partie grâce aux méthodes du capitalisme naissant ${ }^{23}$. Au terme de son étude, Schumpeter porte un jugement optimiste sur l'avenir : l'évolution du monde moderne tendrait à long terme à anéantir les «éléments précapitalistes insérés dans notre vie sociale» et avec eux leurs «potentialités belliqueuses».

En résumé, pour l'auteur «l'impérialisme repose sur un atavisme. Il exprime des rapports de production caractéristiques de modes de production appartenant au passé» ${ }^{24}$. Une telle thèse nous paraît tout à fait recevable s'il s'agit de caractériser la phase d'expansion territoriale de l'Etat national et son échec. Mais sa validité nous semble se borner à cette phase historique étroitement circonscrite. La thèse de Schumpeter qui répand une conception très

\footnotetext{
${ }^{18}$ J. Schumpeter [1919], étude citée, p.118

${ }^{19}$ Ibidem, p.123

${ }^{20}$ Ibid., p. 131.

${ }^{21}$ Ibidem, p. 145.

${ }^{22}$ Ibid., p. 145.

${ }^{23}$ Ibid., J. Schumpeter ajoute : « II n'aurait jamais pu naître de la logique interne du capitalisme lui-même. Cela est encore vrai de l'exportation monopolistique qui vient en droite ligne de la politique de la monarchie absolue et des habitudes commerciales particulières à un milieu fondamentalement précapitaliste (p. 151-152)»

${ }^{24}$ Ibid., p. 110. J. Schumpeter ajoute : «II constitue un élément atavique tant dans les structures sociales que dans les catégories affectives de la personnalité.» Ce sont ces catégories affectives qui entretiennent la persistance de représentations collectives et de politiques périmées.
} 
étroite de l'impérialisme nous semble laisser totalement inexpliqués les phénomènes contemporains d'emprise de structure ou d'annexion économique pacifique qui attestent la permanence et la vigueur de ces relations de pouvoir économique, qui « sont manifestes dans les luttes- concours qui se déroulent entre agents inégaux et entre groupes organisés». La main invisible qui exprime la rationalité du système capitaliste, selon Schumpeter, n'a décidément pas réussi à exorciser «les pouvoirs indiscernables» qui participent de l'essence même du système capitaliste.

\section{Deux types différents d'interprétation économique de l'histoire}

Comment identifier et comparer les éléments essentiels des méthodologies mises en œuvre par Hicks et Schumpeter dans leurs analyses historiques ? Hicks opère un renversement de l'opposition classique entre analyse statique et analyse dynamique, pour retrouver par différence avec les conditions de la formation d'un équilibre statique de type walrasien, les présupposés logiques et historiques de l'économie marchande. Pour ce qui concerne les présupposés logiques, Hicks, au lieu de s'enfermer dans une analyse dynamique qui s'intéresserait à l'évolution des phénomènes sociaux globaux au cours du temps, va les découvrir en réduisant ces phénomènes sociaux aux actions individuelles qui les composent et en montrant comment celles-ci s'inscrivent dans un processus historique dont le résultat est précisément la réalisation des conditions de l'équilibre statique, c'est-à-dire de ces données que le chercheur pose comme allant de soi, au point de départ de sa réflexion.

Dans Valeur et Capital, Hicks écrit (p. 21) que « l'économie pure ressemble fort à un tour de passe-passe ; on a l'air de vous présenter une proposition a priori ayant trait à la réalité. C'est un jeu fascinant que d'essayer de trouver l'astuce, car à moins d'être parfaitement crédule, astuce il y a. (...) La première consiste à admettre au début d'un raisonnement que les seules choses qui importent dans un problème pratique sont celles qui interviennent dans le raisonnement. " $\mathrm{Au}$ fond, Hicks adopte une conception purement instrumentaliste de l'équilibre walrasien, en tout point comparable à celle développée par Schumpeter dans son ouvrage de 1908. Cette conception conduit à distinguer deux catégories d'hypothèses : des hypothèses théoriques qui n'ont aucune traduction dans la réalité puisqu'elles représentent des artifices méthodologiques destinés à développer un raisonnement portant sur des concepts abstraits; des hypothèses historiques qui tentent d'exprimer les éléments singuliers des phénomènes réels, qui peuvent être vérifiées, et qui présentent un aspect cognitif puisqu'elles nous informent sur la manière dont les choses se passent. Dans sa théorie de l'histoire économique, Hicks, et c'est évidemment une performance remarquable (et peu remarquée!) utilise un nombre extrêmement restreint d'hypothèses historiques. Au point que les carences que nous avons relevées dans son analyse sont le plus souvent l'expression d'une absence, précisément l'absence d'une hypothèse historique pertinente.

Mais le projet de Hicks justifie en quelque sorte cette absence d'hypothèse historique. Son souci constant est plutôt d'identifier les institutions tels les contrats, qui en assurant une homogénéisation des comportements reproduisent dans la réalité les effets que l'analyse déduit des hypothèses théoriques nécessaires à la démonstration de la stabilité de l'équilibre statique. En d'autres termes, Hicks développe une théorie structuraliste de l'histoire dont la signification serait la suivante : la saisie d'un mouvement quel qu'il soit nécessite le recours à un référentiel. Or la détermination des conditions d'un équilibre statique permet de repérer ce qui dans un système économique peut être considéré comme invariant dans le temps. Précisément, l'énoncé des conditions de l'équilibre statique permet de caractériser les propriétés relatives à l'organisation du système économique, qui sont invariantes dans le 
temps. L.H. Dupriez [1947] a relevé à juste titre que, du point de vue de la théorie économique, l'analyse statique devait être considérée comme plus générale et donc plus fondamentale que l'analyse dynamique. L'objet de l'analyse dynamique, qui est de découvrir les conséquences des modifications ayant affecté les relations entre éléments du système économique, est en un sens limité : ce ne sont pas les variations des grandeurs économiques qui créent la solidarité des instants; celles-ci ne font que manifester les étapes du déroulement d'un processus historique dont l'explication renvoie à l'analyse des lois de fonctionnement du système économique. En d'autres termes, l'irruption de l'histoire est canalisée dans une analyse sous forme de périodes, où les enchaînements sont conçus comme indépendants du processus historique lui-même. L'histoire dans cette perspective n'est pas cumulative : il n'y a ni apprentissage, ni répétition, mais reproduction à travers l'infinie variété des formes historiques d'un ensemble déterminé de rapports qui constituent ce que l'on peut appeler avec Hicks l'économie marchande. La théorie de l'histoire de sir John Hicks accepte ainsi « la priorité absolue du point de vue synchronique» et peut être en ce sens qualifiée de structuraliste.

L'analyse historique de Schumpeter procède directement de la théorie mengerienne, en prenant pour point de départ une définition préalable d'une catégorie de faits sociaux, «conçue comme la mise en forme logique d'un découpage opéré par le sens commun, dont on peut étudier à l'état presque pur les effets et la manière d'opérer» (J.-C. Passeron [1972,], p. 22). A partir de cette définition préalable, et en suivant le précepte de " l'économie de raisonnement», emprunté à Mach, J. Schumpeter, en formulant un certain nombre d'hypothèses historiques relatives à la signification de phénomènes comme le nationalisme et l'exportation monopolistique, essaie de démontrer que ces phénomènes liés historiquement au développement du capitalisme, n'en sont en aucune façon les effets puisque étrangers à la logique de fonctionnement du capitalisme pur.

Cette démonstration permet d'établir la distinction essentielle entre les caractères de la dynamique économique du capitalisme, qui en manifestent les propriétés de l'évolution en tant que système de rapports économiques déterminés, et les attributs singuliers d'une évolution historique particulière qui greffe sur les premières des déterminations d'une autre nature. En effet, il n'est pas suffisant de comprendre la façon dont un phénomène social, tel que l'impérialisme, s'est développé pour en dégager la logique interne. Une véritable analyse historique appliquée, d'une part, à l'étude du phénomène impérialiste et, d'autre part, à l'analyse de l'évolution du système économique capitaliste, devrait procéder de la manière suivante : il conviendrait tout d'abord de se garder d'appliquer des concepts (exemple : le profit) issus de situations sociales déterminées, à des époques où les structures fondamentales des systèmes sociaux sont foncièrement différentes et, ensuite, il conviendrait d'apprécier les situations historiques dans leur totalité. «C'est, en tant que totalité, — précise, en effet, J. Schumpeter - (et non en tant qu'abstraction) qu'une situation concrète produit la situation qui lui succédera. Mais le système, tel qu'on peut le dégager abstraitement, ne produira pas nécessairement un système possédant la même logique, étant donné que les éléments historiques que l'on peut y appréhender objectivement ne représentent, dans le meilleur des cas, qu'une partie des causes agissantes. C'est là une des difficultés inhérentes à toute théorie de l'évolution» ${ }^{25}$.

\footnotetext{
${ }^{25}$ J. Schumpeter [1918] : La crise de l'Etat fiscal, reproduit in Impérialisme et classes sociales, op. cit., p.238.
} 
Il apparaît ainsi que Schumpeter applique de manière réflexive, à l'utilisation de l'analyse économique à des fins d'interprétation historique, sa distinction entre éléments relevant d'une connaissance scientifique et éléments relevant d'un discours idéologique. En d'autres termes, les exigences méthodologiques de l'analyse historique conduisent à qualifier d'idéologiques (et l'on songe évidemment irrésistiblement ici à tous ces faits stylisés dont sont encombrés les manuels les mieux intentionnés), les applications de l'analyse économique au matériau historique, qui conduiraient à déduire, des attributs théoriques du type d'analyse économique employé, la signification des processus historiques étudiés.

Il convient de trouver les raisons des différences manifestes entre ces deux pratiques de l'histoire économique, et plus encore des écarts substantiels entre les préceptes méthodologiques dont elles s'inspirent. Ces raisons renvoient à l'opposition entre une conception moniste des formes de la connaissance qui n'établit pas de différence de nature entre les méthodes des sciences sociales et les méthodes des sciences de la nature et une conception dualiste, qui s'intéressant plus à l'homme qu'aux attributs des faits sociaux, établit entre les sciences sociales et les sciences de la nature une discontinuité irréductible.

\section{Monisme ou dualisme dans les formes de la connaissance : les véritables enjeux des rencontres entre économistes et historiens.}

Indépendamment de ses incursions dans les débats méthodologiques contemporains (cf. Hicks [1979]), la manière dont Hicks rend compte de sa pratique scientifique tend à l'inscrire très nettement dans une filiation walrasienne. D'une part, il considère l'économie comme une science sociale: « une variété particulière de science sociale qui a trait aux actions 
rationnelles des êtres humains et à leurs conséquences» (Hicks, [1974] p.307) et d'autre part, dans le «manifeste» dont il fait précéder en 1959 ses Essays in world economics il déclare simultanément qu'il abandonne l'idéal de neutralité des partisans de l'économie du bien- être, et que la tâche fondamentale de l'économiste consiste à assumer la responsabilité des conséquences des recommandations en matière de politique économique qui débordent le champ de l'économie et peuvent concerner toutes les sphères de la vie sociale (Hicks [1959], p. XI). Ce faisant, il retrouve la position fondamentale de Walras qui considère que l'économie est une science sociale dont la tâche ne s'achève que lorsque les critères de l'économie pure, de l'économie appliquée et de l'économie sociale ont pu être établis et que leur mutuelle compatibilité a été rationnellement démontrée : «La justice se confirme par son accord avec l'intérêt; l'intérêt rationnel se confirme par l'expérience; et jusqu'à cette confirmation, la science n'est pas définitivement faite» (Walras, [1897], p. 1022). Si Walras lui-même déclare s'inspirer principalement de L.Vacherot [1858] dans l'élaboration de sa distinction entre économie pure, appliquée et science sociale, tant E. Kauder ([1953], p. 572 note 2) que K.Pribram [1986] découvrent dans la manière dont cette distinction est philosophiquement fondée, l'influence de Descartes et d'A. Comte. Nous avons ici, d'une part, une tradition walrasienne de l'unité de la connaissance scientifique, affirmant que les phénomènes sociaux relèvent des mêmes méthodes d'analyse que les phénomènes naturels, et dont les effets se font sentir jusque dans la pratique scientifique de Hicks, et, d'autre part, une synthèse schumpétérienne (cf. N. De Vecchi [1986]) des éléments essentiels des systèmes de pensée de $\mathrm{C}$ Menger et de $\mathrm{M}$. Weber, qui règle les rapports économie-histoire sur la base d'une reconnaissance de la spécificité des sciences sociales et de leurs méthodes.

La querelle des méthodes qui trouve sa source dans un état philosophique aux ramifications complexes et dont les tenants et aboutissants ont été minutieusement analysés par M. Weber [1903, 1905, 1906] reste largement ignorée par le courant de l'économie mathématique ainsi qu'en témoigne en particulier la correspondance entre L. Walras et C Menger (W. Jaffé [1965], vol. 2). Néanmoins, comme le montre O. Neurath [1910] les mêmes problèmes apparaissent dans les travaux des tenants de l'économie mathématique et dans ceux des protagonistes de la querelle des méthodes. Ces problèmes sont relatifs, d'une part, aux critères d'une connaissance scientifique, d'autre part à la spécificité ou non des sciences sociales, enfin aux rapports de la connaissance scientifique à la réalité qu'elle est censée rendre intelligible sinon expliquer.

Pour tenter de résoudre ces problèmes, deux stratégies sont mises en œuvre. L'une, est celle qu'élabore C. Menger en fournissant les fondements théoriques d'une classification des connaissances : cette stratégie débouche sur la reconnaissance de la spécificité des sciences sociales et sur une épistémologie particulière explicitant les rapports de la connaissance scientifique à la réalité sociale. L'autre stratégie, est celle de Walras, dont $\mathrm{O}$. Neurath trouve les prémisses chez J.-B. Say et A. Comte, qui traite de l'ensemble des questions soulevées sur la base de critères internes à la science économique fondant la tripartition économie pure, économie appliquée, économie sociale et réglant, par là-même, la question des chevauchements d'objet et des frontières des disciplines dans l'analyse des faits sociaux.

\section{L'idéal d'une science unifiée et la négation de la spécificité des sciences sociales}

En matière de faits sociaux, L. Walras professe une théorie de la connaissance qui présente un triple caractère : elle est moniste, naturaliste, et a-historique. Walras considère tout d'abord les phénomènes sociaux comme des phénomènes naturels : la société, écrit-il, est 
un « fait naturel et nécessaire et non point conventionnel et libre» ${ }^{26}$. Cette thèse qui trouverait son origine dans les écrits philosophiques de E. Vacherot [1858] incite Walras à prêter une attention particulière aux doctrines philosophiques qui entendent faire la synthèse entre le matérialisme et le spiritualisme. C'est ainsi que l'ouvrage Le Monisme de l'Allemand Haeckel publié en français en 1897 est remarqué par L. Walras dès son apparition. «Ce naturaliste généralise la théorie darwinienne du transformisme en l'appliquant aux phénomènes de l'esprit, qui dériveraient des sensations au même titre que les phénomènes forces ou que les phénomènes matière, les choses et les êtres ne relevant que d'un seul élément constitutif» écrit F. Bompaire en 1931 dans son examen des fondements philosophiques de la pensée de Walras $^{27}$.

Dans l'œuvre de Haeckel, Walras trouve la confirmation de sa deuxième thèse qui affirme l'identité des critères de vérité qu'il s'agisse de la connaissance des faits de la nature ou de la connaissance des faits moraux. Enfin, la troisième caractéristique de la théorie walrasienne de la connaissance des faits sociaux réside dans le refus affirmé de transposer aux faits sociaux la méthode d'isolement des sciences de la nature : «La mécanique, la physique, la chimie, la physiologie expérimentent. En raison de la nature impersonnelle des faits qu'elles étudient, elles peuvent isoler certains d'entre ces faits et les faire apparaître, cent fois sur cent, dans un rapport que, par induction, elles sont fondées à considérer comme une loi physique ou naturelle. L'économie politique n'expérimente pas ; elle observe des faits personnels toujours amalgamés les uns avec les autres et dont elle ne peut jamais dire avec certitude comment ils se sont produits. D'où il suit que la déduction doit toujours venir au secours de l'induction» ${ }^{28}$.

Enfin l'économie politique en tant que connaissance scientifique des faits sociaux considère l'histoire comme un réservoir de faits, à la manière d'A. Comte : «L'histoire nous est infiniment précieuse, et nous ne saurions trop remercier l'école allemande et l'école française de Le Play de nous avoir donné l'histoire économique que nous autres, économistes proprement dits, désireux d'emprunter soit des points de départ, soit des confirmations à l'expérience, nous demandions en vain aux historiens de profession, toujours confinés dans l'histoire politique et militaire» ${ }^{29}$.

En d'autres termes, il y a pour L. Walras, en dépit de la différence de nature entre faits moraux, où règne le libre-arbitre humain, et faits naturels, où règne la nécessité, une identité de méthode dans la connaissance scientifique qui conduit, en faisant abstraction de l'essence des choses, à mettre au jour des relations entre les phénomènes dont le caractère de permanence leur confère le statut de lois, mais dont la signification dépend évidemment des propriétés de l'objet étudié. A cet égard, tant E. Durkheim que G. Schmoller ne disent pas autre chose lorsqu'ils affirment que la découverte des lois en science sociale a trait à la mise en évidence de rapports constants de coexistence ou de succession des phénomènes considérés indépendamment de leur essence : «Traiter les faits d'un certain ordre comme des choses, ce n'est donc pas les classer dans telle ou telle catégorie du réel ; c'est observer vis-àvis d'eux une certaine attitude mentale. C'est en aborder l'étude en prenant pour principe qu'on ignore absolument ce qu'ils sont et que leurs propriétés caractéristiques, comme les causes inconnues dont elles dépendent, ne peuvent être découvertes par l'introspection même de la

\footnotetext{
${ }^{26}$ L. Walras [1986]: Recherche de l'idéal social, sixième leçon in Etudes d'économie sociale, œuvres économiques complètes d'Auguste et Léon Walras, tome IX, Economica, [1990], p. 129.

${ }^{27}$ F. Bompaire [1931] : Du principe de liberté économique dans l'œuvre de Cournot et dans celle de l'Ecole de Lausanne, Thèse, Université de Poitiers, Sirey, p. 485.

${ }^{28}$ L. Walras [1897], p. 1022-1023.

${ }^{29}$ Ibidem, p. 1023.
} 
plus attentive», Durkheim ([1901], p. 5). L'attitude de principe à laquelle Durkheim entend se tenir est exactement celle qu'adopte G. Schmoller comme l'observe J.Schumpeter: «il y avait chez lui le respect du fait économique et la volonté de le laisser parler de lui-même» ([1983], p. 90). J. Schumpeter ajoute que l'acception scientifique de l'étiquette historico-éthique que G. Schmoller apposait à son école avait précisément pour fonction de rappeler, qu'à l'opposé de la «méthode d'isolement» prônée par C.Menger sur la base d'une théorie de l'essence économique des éléments structurants de l'activité économique, méthode et théorie véhiculant aux yeux de G. Schmoller des jugements a priori arbitraires, il convenait «d'étudier toutes les facettes d'un phénomène économique; partant toutes les facettes du comportement économique et pas seulement la logique économique de ce comportement; partant l'ensemble des motivations humaines telles qu'elles se manifestent historiquement» ([1983], p. 92).

Cet ensemble de règles de conduite scientifique devrait nous rendre sensibles au double enracinement, dans une théorie de la connaissance à la fois positive et moniste du programme de recherche schmollerien, qui le situe dans un rapport de proximité beaucoup plus grand à l'œuvre de L.Walras qu'à celle de C. Menger. F. Simiand, dont Schumpeter ([1983], p. 102) nous rappelle qu'il dirige contre la théorie traditionnelle les mêmes arguments que G. Schmoller et dont la méthode serait «institutionnaliste» ([1983], p. 102, note 3) ne s'y est point trompé :

«II est, je crois, nettement inexact de considérer que l'école dite historique ne pense pas aboutir à des lois. (Voir la Préface du $2^{\text {ème }}$ volume du Grundriss de Schmoller où il s'oppose lui-même autant aux historiens purs qu'aux économistes orthodoxes et le chapitre méthodologique de ce même précis, I, pp. 99-111). Sans doute les lois auxquelles les économistes arrivent ou peuvent arriver ne sont pas universelles en ce sens qu'elles exprimeraient la vie économique de tous les temps et de tous les pays : ce sont des lois d'évolution et des lois relatives,: mais apporter la notion d'évolution dans une matière à science expérimentale n'est pas renoncer à la science de cette matière,; tout au contraire. La distinction conforme à la division réelle des économistes serait plutôt une distinction entre la tendance à une science conceptuelle, idéologique d'une part, et la tendance à une science positive, expérimentale, d'autre part. Mais du reste, il est rare qu'aucune de ces deux tendances soit pure et soutenue jusqu'au bout dans aucune des écoles passées » (in Lalande, [1926], 15e édition, [1985], pp. 262-263).

Si les formes de la connaissance scientifique sont ainsi réputées identiques, en dépit de la spécificité des objets de connaissance, qu'apporte la connaissance des faits historiques à l'économie politique? C'est sur ce point que s'opère le clivage entre les théoriciens de l'économie pure et ceux de l'école historique allemande. Walras, dont la pensée fut influencée par celle d'A. Comte, est sur ce point justiciable de la critique formulée par J. Schumpeter : «on pourrait qualifier de comtiste l'idée que l'histoire est source de faits. Or Comte allait à cette source (ou nous conseillait d'y aller) en vue de découvrir — par une méthode qu'il croyait identique à celle qu'on suit dans les sciences naturelles — des "lois historiques". Le dessein scientifique de Schmoller était tout différent. Pour lui, la suggestion de Comte était l'incarnation même de "l'erreur naturaliste" et les lois historiques de Comte était des impostures» (J. Schumpeter [1983], p. 91).

En fait, il y a une distinction dans l'œuvre de Schmoller entre la connaissance scientifique, qui est une et dont relève l'économie politique, et la connaissance historique qui constitue la matrice de la précédente en ce qu'elle définit l'identité historique des faits sociaux. Beaucoup plus proches des analyses de l'école historique du droit de Savigny, que des thèses 
kantiennes ou néo-kantiennes ${ }^{30}$, les théories de G. Schmoller quant au contenu de la connaissance historique, affirment que le droit, comme les langues et les mœurs sont le produit d'une création collective, inconsciente et involontaire et qu'il n'est donc pas possible de comprendre ces institutions et de les interpréter autrement que par une étude historique. Ainsi, «L'ancienne économie politique orthodoxe considérait, à son point de vue technologique et individualiste, la division du travail comme une espèce de prodige, comme une harmonie préétablie à laquelle les individus, qu'elle considère comme indépendants et isolés, se soumettent quasi-inconsciemment, et presque sans avoir à faire acte de volonté, invinciblement entraînés par les avantages de l'échange» (G. Schmoller [1890], p. 240). Or, poursuit G. Schmoller, la division du travail « considéré dans son ensemble comme un procès historique dépendant des mœurs, du droit et de l'organisation sociale», «réclame la fixation de certains usages relatifs aux échanges, aux paiements, aux obligations réciproques, qui, en général, prennent naissance sur des espaces restreints et se répandent plus loin sous des formes fixes et arrêtées. Ces usages engendrent certaines institutions qui représentent dans notre état social comme le précipité des frictions et des luttes antérieures (...) (G. Schmoller [1890], p. 238).

\section{Le statut singulier des sciences de l'économie et les fondements de leur autonomie}

C. Menger élabore sa réflexion sur les fondements des sciences sociales à partir des mêmes sources que G. Schmoller, à savoir l'œuvre de Savigny. Mais le contexte philosophique dans lequel chacune de ces œuvres se déploie est radicalement différent. En Allemagne, le refus d'appliquer à l'étude des sciences sociales les méthodes des sciences physiques trouve son origine dans l'idée kantienne selon laquelle «les lois de causalité ne sont pas applicables à l'analyse du comportement humain parce qu'elles sont incompatibles avec le libre-arbitre» (Pribram, [1986], p. 213).

Cette idée introduit deux principes à propos desquels se cristallisent les débats de méthode entre l'école historique allemande et l'école de Vienne. Le premier principe est celui du nécessaire recours à des processus intuitifs pour l'analyse des phénomènes sociaux (principe que retiendra encore M. Weber, contre la méthode d'isolement), processus «qui permettent aux observateurs de comprendre la nature et le fonctionnement d' "ensembles" collectifs intégrés qui, bien que non accessibles à la perception directe, peuvent être considérés comme des objets ayant une existence réelle en dehors de l'esprit humain» (Pribram [1986], p. 213). Le second principe, que l'on peut qualifier de principe de sélection a pour fonction de régler le problème au cœur de la querelle des méthodes selon O. Neurath, à savoir, comment les différents chercheurs en science sociale qui traitent des mêmes phénomènes sociaux conçoivent-ils les rapports entre les aspects dont ils traitent et ceux dont ils ne traitent pas. A la suite des critiques formulées par H. Lotze à la théorie kantienne de la connaissance, Windelbandt puis Rickert développent une nouvelle orientation philosophique selon laquelle, d'une part la question de la validité d'un énoncé est désormais circonscrite à celle des règles de cohérence entre les concepts, indépendamment de toute référence à l'expérience et aux réalités que ces concepts entendent désigner (cf. S. Parsons, [1990]); d'autre part, la référence aux valeurs est introduite comme la forme d'existence du critère de sélection dans les sciences sociales, qui entend distinguer au regard des préoccupations spécifiques de ces disciplines ce qui est essentiel et ce qui ne l'est pas.

\footnotetext{
${ }^{30}$ Toutefois, dans ses derniers écrits sur la méthode, G. Schmoller leur reconnaît un rôle appréciable. Cf. G. Schmoller "Volkswirtschaft, Volkswirtschaftslehre und Methode", in Handwörterbuch der Staatswissenschaften, 3. Aufl., 8, bd, Jena 1911.
} 
S'agissant des sciences historiques, H. Rickert affirme dans son article consacré aux Quatre modes de l' "Universel" dans l'histoire "qu'il ne peut être question de nier que toute espèce d'exposé historique présuppose une certaine détermination des valeurs à laquelle il se réfère, pour déceler quels événements seront, à ses yeux, essentiels ou non» ${ }^{31}$. Il précise plus loin que: "les éléments ultimes, qui servent à construire tout exposé historique, sont nécessairement universels; mais tandis que les sciences naturelles n'emploient ces éléments qu'à la formation de concepts également universels; l'histoire les emploie à construire une cuvre à contenu individuel. Nous avons vu, en second lieu, que l'histoire ne peut pas exprimer tous les phénomènes individuels, et qu'elle se borne à ceux qui ont une portée universelle ; mais cette portée universelle ne vient pas de ce que les événements seraient subsumés à titre d'exemplaires sous un concept général ; elle vient précisément du caractère propre et individuel de ces événements. Nous avons vu, en troisième lieu, que l'histoire n'isole pas ces objets, mais qu'elle les ordonne en un complexus général ; d'ailleurs, ce complexus général n'est en aucune manière un concept à contenu universel, sous lequel les objets seraient subsumés ; il est un tout constitué par les diverses parties individuelles et il forme lui-même une réalité individuelle que l'on peut sans doute considérer comme plus vaste, mais non comme plus universelle que ses parties. Ainsi dans chacun de ces trois cas, ou bien l'Universel historique n'est en aucune façon un concept général, comme ceux que construisent les sciences naturelles; ou bien, lorsqu'il est vraiment un concept général, il sert seulement de moyen pour exprimer l'individuel et le particulier» (H. Rickert [1901], p. 135).

Face à cette dissidence philosophique néo-kantienne, qui sera en quelque sorte inoculée dans le programme de recherche schmollerien par M. Weber, la philosophie aristotélicienne de la connaissance dont s'inspire C Menger (cf. E. Kauder [1962], Cubeddu [1985], B. Smith [1986] ) détermine une reconnaissance de la spécificité des sciences sociales sur la base d'un principe d'explication génétique, opposé à la connaissance intuitive; d'une méthode synthétique («compositive method») qui, partant de la décomposition d'un phénomène complexe en éléments essentiels (et non simples!), entend reconstruire l'ensemble initial sur la base du maniement d'entités constamment intelligibles, enfin et surtout d'une conception réaliste, inspirée de l'enseignement philosophique de F. Ueberweg, qui affirme que les concepts ne sont que la traduction symbolique d'une réalité préexistante dont les principes de fonctionnement peuvent être mis au jour par une compréhension fondée sur un rapport de familiarité avec le système de relations existant entre les phénomènes et qui est un effet de leur essence. S'agissant de l'économie, cette conception de la nature des sciences sociales induit une autre formalisation du rapport individu-institution et du rapport aux réalités historiques que celle retenue par l'école historique allemande, y compris dans sa reformulation wéberienne.

En s 'inspirant des relations établies par Aristote entre les catégories de forme et de matière, l'achèvement d'une forme traduisant le développement des propriétés inhérentes à la matière, C. Menger fonde le nécessaire recours à une explication de type génétique (Kauder, [1957] ) , puisqu'il convient de remonter de ces propriétés intrinsèques des phénomènes telles que les constate la statistique et l'investigation historique, aux lois «fixes» qui régissent l'accomplissement immuable des potentialités de la matière. On comprend, de ce point de vue, que les individus n'aient qu'une perception limitée et en quelque sorte informe du milieu naturel et institutionnel au sein duquel ils agissent. Les institutions elles-mêmes ont, pour ces raisons, une origine «organique» (cf. De Vecchi [1986], Cubeddu [1985]) : elles sont certes

\footnotetext{
${ }^{31}$ H. Rickert [1901]: Les quatre modes de «l'Universel » dans l'histoire, Revue de synthèse historique, vol. 2, n ${ }^{\circ} 5$, p. 134.
} 
le sous-produit des interactions individuelles, mais sans que l'on puisse assigner à celles-ci «comme le veut le rationalisme abstrait, une origine intentionnelle qui reviendrait à assujettir l'agir individuel à des critères de rationalité indépendants de la connaissance limitée que chaque individu a du contexte dans lequel il opère» (De Vecchi [1986], p. 6).

Ainsi, au cœur de la problématique mengerienne, se situe le problème du processus social qui confère aux relations entre les interactions individuelles et les institutions des caractéristiques déterminées $^{32}$. C'est la raison pour laquelle Menger adopte une méthode synthétique, irréductible à l'individualisme méthodologique, puisque le recours à l'intentionnalité des agents lui paraît insuffisant. Pour lui, le problème essentiel de l'économie théorique consiste dans la compréhension du processus qui fait advenir de la convergence d'actions inintentionnelles, des institutions, sorte d'instances régulatrices des comportements individuels. Il n'est, à ses yeux, nul besoin pour interpréter la signification de ces institutions, de recourir à des normes de comportement : il s'agit bien, au contraire, de découvrir le soubassement rationnel à des conduites humaines qui laissent apparaître derrière la multiplicité des situations concrètes, des systèmes de références fixes. A l'opposé du rationalisme «pragmatique» d'un Smith, cette méthode synthétique tente fondamentalement d'expliciter les articulations entre individu et société sur la base de la reconnaissance préalable d'une détermination de la nature et des caractères des agents individuels par leur existence en société (Hayek [1948], p. 6). Ainsi la dialectique entre un contexte socialement déterminé et une logique de l'agir individuel subsumée sous l'action régulatrice d'institutions, dont l'origine est à rechercher dans une répartition arbitraire des connaissances et des informations, conduit à l'idée d'une économie, comme science sociale spécifique. Cette science, aurait la particularité de voir la validité de son enseignement théorique, en termes de recherche de principes d'organisation sociale, dépendre du recours scientifiquement contrôlé à des disciplines auxiliaires, parmi lesquelles la statistique et l'histoire figurent au premier plan sans pour autant perdre leur identité, qui dans la tradition allemande disparaîtrait, voire serait anéantie sous les propriétés génériques des sciences dites, selon les cas, historiques, de l'esprit ou de la culture.

Les deux systèmes de pensée règlent de façon tout à fait antinomique les rapports de l'économie à l'histoire, à l'occasion de leur analyse du lien des énoncés théoriques à la réalité sociale. Il est à cet égard, très révélateur, que L. Walras considère l'histoire comme un système de faits servant au contrôle de la pertinence empirique des énoncés théoriques. Les critères de la tripartition entre économie pure, économie appliquée et économie sociale le manifestent clairement. Tandis que l'économie pure, en tant que « science pure morale qui constate des faits et des rapports prenant leur source dans la volonté libre de l'homme» est essentiellement a-historique, l'économie appliquée, qui énonce «des règles pour la conduite des personnes les unes vis-à-vis des autres » ne peut le faire que dans un cadre historique et institutionnel dûment spécifié, puisque la recherche du juste, à laquelle doivent être nécessairement assujettis les principes d'organisation de l'économie appliquée, revêt une signification essentiellement contingente. Selon F. Bompaire dans le système walrasien «la politique économique ne considère que des situations essentiellement passagères»; toute réforme sociale même la plus sérieuse et la plus durable «est une transaction entre les conditions d'un point de départ et celles d'un but où l'on veut arriver» (Walras, Economie appliquée, pp. 455-456).

La position de C. Menger paraît beaucoup plus élaborée. Il réfute d'abord l'idéal d'une science universelle de l'économie. Pour lui les sciences économiques appartiennent à quatre

\footnotetext{
${ }^{32}$ C Menger [1883] : Die Irrtumer der Historismus, p. 43.
} 
catégories, qui ont chacune leurs propres critères de validité, à savoir les sciences historiques de l'économie, essentiellement la statistique et l'histoire économique; les sciences morphologiques des phénomènes économiques qui ont pour objet l'énoncé des relations entre la nature des phénomènes singuliers et leur prise en compte dans une synthèse qui procède par isolement et donc qui sélectionne selon des caractéristiques génériques ; la théorie pure des phénomènes économiques; et enfin les sciences pratiques de l'économie. Cette classification découle d'une vision globale des principes logiques qui régissent l'articulation des différentes dimensions des phénomènes économiques ${ }^{33}$, vision globale qui justifie l'élaboration d'une classification des sciences mais non d'une synthèse puisque les connaissances issues de ces différentes sciences sont le produit d'objectifs et de motivations spécifiques et inconciliables. Le statut de science auxiliaire que revêt l'histoire dans ce système de pensée, s'il n'est pas un statut mineur, renvoie cependant à une compréhension appauvrie des exigences d'une pensée de l'évolution.

En tentant une synthèse de la compréhension wéberienne des processus d'évolution et de la méthodologie mengerienne d'élucidation des principes d'intelligibilité des phénomènes sociaux, J. Schumpeter peut être reconnu comme le véritable fondateur du projet d'une économie historique, dont F. Braudel [1950] réactualisera la nécessité théorique et les impératifs méthodologiques ${ }^{34}$.

La théorie schumpétérienne de l'évolution réconcilie l'irruption de phénomènes singuliers — qui ne peuvent être intelligibles qu'en termes d'une logique de l'agir humain tels que la substitution de nouveaux critères de choix à ceux auxquels les agents se fient habituellement pour prendre leur décision, et l'endogènéisation de ces facteurs dynamiques à l'aide des propriétés de l'organisation du système économique global, en l'occurrence l'institution du crédit bancaire, qui, en mettant à disposition de l'entrepreneur-innovateur un pouvoir d'acquisition, décerne à celui-ci et à son projet un certificat d'acceptabilité sociale (Dufourt [1990]). On retrouve ici, comme l'a nettement souligné N. de Vecchi [1986] la thématique mengerienne de l'interaction entre l'agir individuel et le fonctionnement des institutions sociales. C'est cette interaction qui rend compatible le changement des règles à partir desquelles les agents effectuent leurs prévisions et l'endogènéisation de ces changements. En outre, si l'on suit J. Schumpeter, et que l'on relève avec lui les caractéristiques fondamentales du procès de "scientifisation" de la connaissance historique (la substitution de l'explication des processus à la simple description des événements; l'emprunt aux sciences sociales des questions pertinentes en vue de détecter des fils conducteurs dans l'écheveau des événements; la référence aux problèmes rencontrés dans l'exercice d'une pratique qu'elle soit scientifique, professionnelle, artistique pour interpréter les conduites) $)^{35}$, il est impossible de ne pas reconnaître la nécessité d'approfondir la différence de contenu et d'ambition scientifique entre une pensée de l'évolution et une analyse des propriétés de la dynamique économique. Essentiellement historique, au sens où elle intégrera les caractéristiques wéberiennes d'une connaissance enracinée dans un système de valeurs, attentive à une compréhension des sens subjectifs des conduites individuelles, et orientée vers une analyse causale « qui comporte à titre de démarche essentielle, la construction de ce qui se serait passé si l'un des antécédents ne s'était pas produit ou avait été autre qu'il n'a été» (R.Aron [1967], p. 515), la pensée schumpétérienne de l'évolution réalise une synthèse, entre causalité historique et causalité économique, l'une et l'autre renvoyant à la conception

\footnotetext{
${ }^{33}$ Cf. C Menger [1889] : Grundzuge einer Klassifikation der Wirtschaftswissenschaften, Conrads Jahrbuch, pp. 465-496.

${ }^{34}$ Voir P. Dockès, B. Rosier [1991] : Histoire « raisonnée» et économie historique, Revue économique, vol. 42 n $\circ 2$.

${ }^{35}$ J. Schumpeter [1927] : Sombarts Dritter Band. Schmollers Jahrbuch, tome 51, vol. I, p. 5.
} 
statistique de l'équilibre qui aurait été en définitive celle de C.Menger (cf. E. Streissler [1988], p. 197).

La récurrence des rencontres entre historiens et économistes renvoie ainsi à une double question, l'une interne à l'économie en tant que discipline scientifique autonome ; l'autre extérieure à cette discipline. La première question s'énonce ainsi : l'économie politique est-elle ou non une science sociale ? La deuxième peut être exprimée sous une forme lapidaire: le rapport à l'histoire constitue- t-il le fondement ultime de la spécificité des sciences sociales?

Que ces deux questions n'aient pas reçu de réponse définitive, on en trouvera la manifestation irrécusable dans le retour de ces «rencontres». Le projet d'une économie historique d'intention scientifique nécessite un retour aux sources et une compréhension des raisons pour lesquelles à partir de la même référence centrale, à savoir la logique de Wundt ${ }^{36}$, il est possible de déduire des arguments pour (cf. C. Menger) et contre (cf. O. Neurath) la spécificité des sciences sociales, et des énoncés contradictoires quant aux formes de la connaissance en économie.

\section{Références}

Andreano R. Ed. [1970] The new economic history, Recent papers on methodology. John Wiley and Sons, New York. Traduction française, précédée par Le dossier de la question par J. Heffer, Gallimard, [1977].

Aron R. [1950] La philosophie de l'histoire in L'activité philosophique contemporaine en France et aux USA, tome II, Presses Universitaires de France.

Aron R. [1967] Les étapes de la pensée sociologique, Gallimard, Paris.

Barraclough G. [1980] Tendances actuelles de l'histoire, Flammarion, Paris.

Bompaire F. [1931] Du principe de liberté économique dans l'œuvre de Cournot et dans celle de l'école de Lausanne, Thèse, Université de Poitiers. Editions Sirey, Paris.

Boyer R. [1989] Economie et histoire : vers de nouvelles alliances, Annales, Economies, Sociétés, Civilisations, vol.44, $\mathrm{n}^{\circ} 6$.

Bouvier J. [1965] L'appareil conceptuel dans l'histoire économique contemporaine, Revue économique, vol.16, $\mathrm{n}^{\circ} 1$.

Braudel F. [1950] Pour une économie historique, Revue économique, vol. 1, $\mathrm{n}^{\circ} 1$.

Cubeddu R. [1985] Fonti Filosofiche degli «Untersuchungen uber die Methode der Sozialwissenchaften » di Cari Menger, Quaderni di storia dell'economia politica, vol. $3, \mathrm{n}^{\circ} 3$.

\footnotetext{
${ }^{36}$ Cf. les commentaires critiques de la Logique de Wundt [1883] par C Menger [1889], pp. 475 et 494.
} 
Dockes P., Rosier B. [1991] Histoire raisonnée et économie historique, Revue économique, volume $42, \mathrm{n}^{\circ} 2$.

Dupriez L.H. [1947] Des mouvements économiques généraux, 2 volumes, Institut de recherches économiques et sociales, Louvain.

Dufourt D. [1990] Théories changeantes et vérités stables dans 1 'œuvre de J. Schumpeter: à propos de l'essence de l'économie théorique in La méthodologie de l'économie théorique et appliquée aujourd'hui. Colloque annuel de l'A.F.S.E., Nathan.

Durkheim E. [1901] De la méthode objective en sociologie, Revue de synthèse historique, tome III, $n^{\circ} 4$.

Falbeck P. [1908] Die volkswirtschaftliche Literatur Skandinaviens im 19 Jahrundert in Mélanges Schmoller, vol. 1, XIX, Leipzig, Duncker et Humblot.

Farnam H.W. [1908] Deutsche-amerikanische Beziehungen in der Volkswirtschaftslehre in Mélanges Schmoller, vol. 1, XVIII, Leipzig, Duncker et Humblot.

Garrouste P., Dufourt D. [1992] Criteria of scientificity and methodology of social sciences : C Menger, L. von Mises and F. Hayek, à paraître in Perspectives in the history of economic thought, R. Hébert (editor), Edward Elgar.

Gide C. [1890] The economic schools and the teaching of political economy in France. Political science quaterly, vol. 4.

Gide C. [1895] Die neuere volkswirtschaftliche Litteratur Frankreichs, Schmollers Jahrbuch. Jahrbuch für Gesetzgebung, Verwaltung und Volkswirtschaft im Deutschen Reich, vol. 17 (1895).

Gide C. [1907] "Economic Literature in France at the Beginning of the Twentieth Century", The Economic Journal, Vol. 17, No. 66, pp. 192-212.

Gide C. [1908] : L'Ecole économique française dans ses rapports avec l'Ecole anglaise et $\Gamma$ Ecole allemande in Mélanges Schmoller, vol. 1, XVI, Leipzig, Duncker et Humblot.

Haeckel E. [1897] : Le Monisme, lien entre la religion et la science, Scheicher Frères.

von Hayek F. [1948] : Individualism and economic order, Londres, Routledge and Kegan.

Hicks J. [1956] : Valeur et capital, Dunod, Paris.

Hicks J. [1959] Préface and a manifesto in Essays in world economics, Oxford, at the Clarendon Press.

Hicks J. [1973] Une théorie de l'histoire économique, Editions du Seuil, Paris.

Hicks J. [1974] Capital controversies: ancient and modem, The American economic review, vol. $64, \mathrm{n}^{\circ} 2$. 
Hicks J. [1979] Causality in economics, New York, Basic Books.

Jaffe W. [1965] Correspondence of Leon Walras and related papers, 3 volumes, Amsterdam, North Holland Publishing Co.

Kauder E. [1953] The retarded acceptance of the marginal utility theory, Quaterly journal of economics, vol. 67.

Kauder E. [1957] Intellectual and political roots of the older Austrian school. Zeitschrift fur National Ôkonomie, vol. 17.

Kauder E. [1962] : Aus Menger nachgelassenen Papieren, Weltwirtschaftliches archiv, vol. $89, \mathrm{n}^{\circ} 2$.

Lalande A. [1926] : Vocabulaire critique et technique de la philosophie, Presses universitaires de France, 15e' édition, [1985], Paris.

Lecuyer P.B. [1978] Bilan et perspectives de la sociologie de la science dans les pays occidentaux, Archives européennes de sociologie, vol. XIX.

Lotze H. [1874] Logik, Drei Bücher vom Denken, vom Untersuchen und Erkennen.

Mc Closkey D.N. 1976] Does the past have useful economics? Journal of economic literature, vol. 14.

Mc Closkey D.N. [1986] Economics as an historical science in W.N. Parker Ed. Economic history and the modern economist, Basil Blacwell, Oxford.

Marchal A. [1950] Economistes et historiens, Revue économique, $\mathrm{n}^{\circ} 1$.

Mélanges Schmoller (a) [1908] Die Entwicklung der deutschen Volkswirtschaftslehre im neunzehnten Jahrhundert, Erster Teil, Leipzig, Verlag von Duncker und Humblot.

Mélanges Schmoller (b) [1938] Gustav von Schmoller und die deutsche geschichtliche Volkswirtschaftslehre, Festgabe zur hundersten Wiederkehr seines Geburtstages. Schmollers Jahrbuch, vol. 62.

C. Menger [1884] Die Irrtumer des Historismus in der deutschen National Ökonomie, Neudruck der Ausgabe Wien [1884], Scientia Verlag Aalen, [1966].

C Menger [1889] : Grundzuge einer Klassifikation der Wirtschaftswissenschaften. Jahrbucher fur National Ôkonomie und Statistik (Conrads Jahrbuch), N.F. vol. XIX.

O. Neurath [1910] : Zur Théorie der Sozialwissenschaften, Schmollers Jahrbuch, tome 34.

S. Parsons [1990] : The philosophical roots of modern Austrian economics: past problems and future prospects, History of political economy, vol. $22, \mathrm{n}^{\circ} 2$.

J.-C. Passeron [1972] Présentation à J. Schumpeter, Impérialisme et classes sociales, les Editions de Minuit, Paris. 
F. Perroux [1973] Pouvoir et économie, Bordas, Paris.

K. Pribram [1986] Les fondements de la pensée économique, Economica, Paris.

H. Rickert [1901] Les quatre modes de l'Universel dans l'histoire, Revue de synthèse historique, tome II, $\mathrm{n}^{\circ} 5$.

G. Schmoller [1890] La division du travail étudiée au point de vue historique, Revue d'économie politique, vol. 4.

G. Schmoller [1905] Luttes de classe et domination de classe, Revue internationale de sociologie, vol 13 .

G. Schmoller [1911] Volkswirtschaft, Volkswirtschaftslehere und Method in Handwörterbuch der Staatswissenschaften, 3. Aufl., 8. Bd. Jena.

J. Schumpeter [1918] La crise de l'état fiscal, in Schumpeter [1972], Impérialisme et classes sociales, Les Editions de Minuit.

J. Schumpeter [1919] Contribution à une sociologie des impérialismes in J. Schumpeter [1972], Impérialisme et classes sociales. Les Editions de Minuit.

J. Schumpeter [1926] Gustav Schmoller und die Probleme von Heute, Schmollers Jahrbuch, tome 50, vol. 1 .

J. Schumpeter [1927] Sombarts dritter Band, Schmollers Jahrbuch, tome 51, vol. 1.

J. Schumpeter [1935] Théorie de l'évolution économique, Dalloz.

J. Schumpeter [1972] Impérialisme et classes sociales, Les Editions de Minuit.

J. Schumpeter [1983] Histoire de l'analyse économique, 3 volumes, Gallimard.

P.R. Senn [1989] What has happened to Gustav Schmoller in English? History of economics society bulletin, vol. $11, \mathrm{n}^{\circ} 2$.

B. Smith [1986] Austrian economics and Austrian philosophy in W. Grassl et B. Smith Eds, Austrian economics. Historical and philosophical background, Croom Helm, London.

A. Spiethoff [1938] Gustav von Schmoller und die anschauliche theorie der Volkswirtschaft, Schmollers Jahrbuch, vol. 62.

E. Streissler [1988] The intellectual and political impact of the Austrian school of economics, History of European ideas, vol. $9 \mathrm{n}^{\circ} 2$.

FF. Ueberweg [1872] Grundriss der Geschichte der Philosophie, 3.Aufl., Berlin.

E. Vacherot [1858] La métaphysique et la science, Paris: F. Chamerot, 2 volumes. 
